\title{
Clinical Validation of Natural Plant Based Skincare Supplement in Healthy Subjects
}

\author{
Dr. Vidyadhar Kumbhar ${ }^{1}$, Dr. Veena Deo ${ }^{2}$, Ankush Chourasia ${ }^{* 2}$, Dr. Gayatri Ganu ${ }^{3}$, Dr. Rupali Mali ${ }^{2}$ \\ ${ }^{1}$ Director, Sunad Ayurveda, Ayurveda Clinic, Pimpri-Chinchwad, Pune, 1, Mumbai Pune Bypass Road, Shridhar \\ Nagar, Landmark: Near Wolf Colony \& Near Devgarh Society, Pune, India \\ ${ }^{2}$ Consultant, Climic Health Pvt. Ltd., 404, Chartered house, Dr. C. H. Street, Mumbai Mumbai City 400002, India \\ ${ }^{3}$ Director, Clinical Research, Mprex Healthcare, 501, Crossroad, Waked, Pune, India
}

"Correspondence author: Ankush Chourasia; chourasia.ankush0@gmail.com

Received 07 December 2020;

Accepted 29 December 2020;

Published 05 January 2021

\begin{abstract}
Background: The growing field of psycho-dermatology is all about the mind-skin connection. It addresses the link between our emotional state, the body's physiological response to our emotional state, and the outcome of this response on our skin, body, health, and well-being. The aim of this clinical trial is to evaluate safety and efficacy of BP-G01 in improving skin health, complexion and skin luster. Material and methods: Total 33 subjects were screened and out them 3 were considered as drop outs and 30 completed subjects were involved in the study. Natural plant based skincare supplement (BP-G01) contains Plant based protein blend like Plant proteins like rice and pea proteins. The glow blend like evening primrose extract, pearl powder, orange, pomegranate, grape seed extract, sea buckthorn extract and tocopherols from plant source. The adaptogenic blend like Ashwagandha, Amla, Tulsi, Mulethi and Turmeric extracts. It was consumed as 90 days' treatment in dose of 20 gm in the morning after breakfast or post workout with $200 \mathrm{ml}$ of water or milk. Natural plant based skincare supplement (BP-G01) improve improved skin luminosity, brightness, radiance and complexion, skin hydration, skin discoloration, dark circles, dark spots from face and neck and skin evenness indicated by MASI scoring for reducing hyper pigmentation, dark spots/ circles and redness and reduction in mild acne counts. VAS for the improvements in skin complexion and evenness. C.L.B.T. imperfections scale. Result: There was $17.08,22.79,32.56$ and $37.96 \%$ reduction in the MASI score at day 15, 30, 60 and day 90 respectively by reducing hyper pigmentation, dark spots/ circles and redness by MASI scoring. MASI acne score at baseline was $9.10 \pm 1.19$ which was decreased throughout the period of treatment by reduction in mild acne counts as per modified MASI scoring. Conclusion: BP-G01 is safe and effective in promoting skin health in healthy adults.
\end{abstract}

Keywords: skin health, well-being, Nutrition, MASI score, CLBT Scale

\section{Introduction}

Beauty comes from the within. The connection between nutrition and skin health has been an interesting research field. Skin damage consists of two clinically and biologically, distinct processes ${ }^{[1]}$. The first is intrinsic skin changes, which represents chronological aging and affects skin in the same pattern it affects all internal organs ${ }^{[2]}$. The second is extrinsic factor influencing skin health, as a result of external factors and environmental influence, mainly chronic sun exposure and ultraviolet (UV) irradiation but also stress, smoking, pollution, sleep deprivation and nutritional defects.

The growing field of psycho-dermatology is all about the mind-skin connection. It addresses the link between our emotional state, the body's physiological response to our emotional state, and the outcome of this response on our skin, body, health, and wellbeing.

Stress hormones can cause destruction on skin. As a result, stress can lead to breakouts, dark circles, irritation, redness, or wrinkles and fine lines. Cortisol spikes which is a major stress related hormone, damage the collagen and elastin that keep skin smooth, firm, and supple.

Prevention is the best and most effective way to work against extrinsic skin aging effects. The best prevention strategy against extrinsic skin damaging factor sis balanced nutrition, use of natural remedies to improve skin health as a lifestyle, including anti-oxidative rich food/ supplement.

Frequently researched antioxidants such as carotenoids, tocopherols and flavonoids, as well as vitamins (A, C, D and E), essential omega-3-fatty acids, collagen, some proteins and lactobacilli have been referred as agents capable of promoting skin health and beauty ${ }^{[3,4]}$.

The investigational product in the current study is a blend of key ingredients as a natural skin care supplement to promote skin health and luster. The aim of this clinical trial is to evaluate safety and efficacy of BP-G01 in improving skin health, complexion and skin luster.

\section{Materials and Methods}


Study design: An open label, single arm, interventional, prospective, Clinical Study.

The subjects along with their guardians/ parents attending outpatient department of study site(s) were screened for eligibility criteria. Screened for the inclusion and exclusion criteria for the said trial. The subjects who fulfilled inclusion criteria and didn't meet any exclusion criteria had selected to participate in trial.

Inclusion Criteria for the study were: Male/ Female of 18 to 40 years, willing to give a written informed consent and come for regular observations, Subject is a healthy adult, Subject with dull complexion, dry/oily skin with/ without uneven texture and with at least 2 hyperpigmentary spots as a result of photo-damage, Mild acne without nodular abscesses, Subject has not participated in a similar clinical investigation in the past three months, Subject who is willing to abstain from using any fairness product, sunscreens, natural/ Ayurvedic treatments or other home remedies and also willing to abstain from undergoing any facial treatments such as facial masks, packs, bleaching, etc. either at home or at beauty parlour during the entire study course. In case of having used fairness product in the near past, should be willing to undergo a wash out period of 1 week, wherein they should abstain from applying any product other than the suggested cleanser on face, and Female subjects of reproductive age group should undergo UPT at time of screening visit.

Exclusion Criteria for the study were: Subject with a known history or present condition of allergic response to any ingredients used in BP-G01, Subject with a medical history of disease or condition or a concurrent illness or pre-existing or dormant dermatologic condition (psoriasis, rosacea, rashes, eczema etc.) that could interfere with the study results, Subject with excessive facial hair or scars, which could interfere with evaluation. Subject with a history of having used a similar product during or since 1 week prior to the scheduled study commencement, Subject who is currently pregnant (as confirmed by the urine pregnancy test) or nursing or contemplating pregnancy during the study course, Subject who has used any systemic medication (antibiotics, oral contraceptive pills, retinoid, steroids, spironolactone or any anti acne medication) within past 4 weeks or any topical facial medication within 2 weeks prior to study commencement, which could compromise the study, and Patient with known history of acute / chronic liver or kidney disease.

Intervention Details: Product Code: BP-G0, Key Ingredients: Plant based protein blend in BP-G01 consist of Pea and rice protein. Plant proteins like rice and pea proteins

The glow blend in BP-G01 consist of evening primrose extract, pearl powder, orange, pomegranate, grape seed extract, sea buckthorn extract and tocopherols from plant source.

The adaptogenic blend in BP-G01 consist of Ashwagandha, Amla, Tulsi, Mulethi and Turmeric extracts. Blend 20 gm of BPG01 powder in $200 \mathrm{ml}$ of cold or room temperature water or milk.

Outcomes Measures: The primary outcomes of the study is to evaluate safety and efficacy of BP-G01 by assessing-Changes in reducing hyper pigmentation, dark spots/ circles and redness by MASI scoring, acne counts as per modified MASI scoring (For mild acne), VAS score for the improvements in skin complexion and evenness by investigator and subject, Global Assessment Grade for improvement in complexion / dark spots on the face and neck by color grading method, and Patient Satisfaction Questionnaire, Hydration and moisture to the skin, Radiance of the skin by clinical scoring using the coloring, luminosity, brightness, and transparency (C.L.B.T.) scale and imperfections scale (dark circles, redness/rosacea, spots, and heterogeneity).

The secondary outcomes of the study were to evaluate safety and efficacy of BP-G01 by Assessing-Changes in subject's self-assessment score with skin questionnaire (8 question scale), Tolerability of Investigational product by assessing adverse events, serious adverse events during the study period, Global evaluation of overall improvement in complexion on the face and neck by investigator and subject, and The digestive behavior after treatment of product.

Visit Schedule: Screening Visit (up to -14 days), Baseline Visit (Day 0), Visit 1 (Day $15 \pm 5$ days), Visit 2 (Day $30 \pm 5$ days), Visit 3 (Day $60 \pm 5$ days), Visit 4 (Day $90 \pm 5$ days).

Procedure: Principle investigator obtained informed consent from subjects. Subject's demographic details were taken. Subject undergone clinical examination. Subject's medical, surgical and treatment history was taken. Subject's current medication if any was noted in the case record from (CRF). Subject's vitals were recorded.

During screening visit and the entire study duration subjects were advised to refrain from antioxidant agents, vitamins, anti-inflammatory drugs, hormones, Nutraceutical, Ayurvedic, Siddha, Unani, herbal /homeopathic medicines.

On baseline visit, subject was recruited in the study if he or she meets all the inclusion criteria. Subjects were asked for occurrence of any adverse event during screening period. Subjects skin assessment and other questionnaire scoring was performed. Subject was asked to wash the face with mild soap provided at study site to remove dust, residue traces etc. to assess skin clinically. The subjects were not allowed to use the cosmetics which from investigator's discretion can affect study.

The same procedure was followed for all visits including last visit. On every visit the subject was provided with the container of investigational product and critically reviewed for adverse events during study duration.

Global assessment of overall improvement was done by on last follow up visit. Palatability and tolerability with dosage compliance was assessed by the investigator from baseline to end of the study. All the subjects were closely monitored for any adverse event starting from baseline visit till the end of the study visit.

\section{Results}

In the present study, 33 subjects were screened. Out of 33 subjects, 3 lost to follow up in the study. 30 subjects were considered evaluable cases at the end of the study.

Out of 30 completed subjects, the mean age of subjects was $24.2 \pm 2.83$ years of females and the mean age of male subjects was $25.38 \pm 1.55$ years. If compared between the groups, the difference was statistically insignificant. Gender wise subject number per group. The ratio of approximately 80:20 for female and male was decided and maintained during recruitment of the study.

\section{Efficacy Assessments}

Assessment of efficacy in reducing hyper pigmentation, dark spots/ circles and redness by MASI scoring:

There was significant $(\mathrm{p}<0.05)$ reduction from day 15 to day 30 . There was significant reduction in MASI score $(\mathrm{p}<0.001)$ at day 60 and 90. MASI score reduction hyper pigmentation, dark spots/ 
circles and redness from Baseline to end of the study and throughout the study were depicted in (Table 1)

Table 1: Assessment of efficacy in reducing hyper pigmentation, dark spots/ circles and redness by MASI scoring

\begin{tabular}{|l|l|l|}
\hline MASI scoring (Mean \pm SD) \\
\hline Duration(Days) & Score & \% Reduction \\
\hline Baseline & $16.28 \pm 3.14$ & - \\
\hline Day 15 & $13.50 \pm 3.27^{*}$ & 17.08 \\
\hline Day 30 & $12.57 \pm 2.93^{*}$ & 22.79 \\
\hline Day 60 & $10.98 \pm 2.77^{* *}$ & 32.56 \\
\hline Day 90 & $10.10 \pm 3.11^{* *}$ & 37.96 \\
\hline
\end{tabular}

Student " $t$ " Test: $P>0.05$ Not Significant; significant $p<0.01$ *, $p<0.001^{* *}$

Assessment of efficacy in reduction in mild acne counts as per modified MASI scoring:

There was significant $(\mathrm{p}<0.05)$ reduction in MASI acne score from day 30 to day 60 . There was significant reduction in MASI score $(\mathrm{p}<0.001)$ at day 90. MASI score reduction in mild acne counts were assess from baseline to end of the study. The results are resented in table 2 .

Table 2: Assessment of efficacy in reduction in acne lesion counts as per modified MASI scoring

\begin{tabular}{|l|l|}
\hline Duration(Days) & Score Mean \pm SD \\
\hline Baseline & $9.10 \pm 1.19$ \\
\hline Day 15 & $8.70 \pm 1.09$ \\
\hline Day 30 & $8.50 \pm 2.10^{*}$ \\
\hline Day 60 & $8.65 \pm 1.89^{*}$ \\
\hline Day 90 & $7.80 \pm 1.61^{* *}$ \\
\hline
\end{tabular}

By Chi Square Test: $P>0.05$ Not Significant; significant $p<$ $0.01 *, p<0.001 * *$

Table 4: Assessment of C.L.B.T. color imperfections scale

\begin{tabular}{|l|l|l|l|}
\hline \multirow{2}{*}{ Duration (Days) } & CLBT SCORE Mean \pm SD $(\mathbf{N}=\mathbf{5 0})$ \\
\cline { 2 - 4 } & $\begin{array}{l}\text { Red discoloration Saturation } \\
(\boldsymbol{\%})\end{array}$ & $\begin{array}{l}\text { Olive discoloration Saturation } \\
\mathbf{( \% )}\end{array}$ & $\begin{array}{l}\text { Beige colored evenness Change from 50\% } \\
\text { saturation }(\boldsymbol{\%})\end{array}$ \\
\hline Baseline & $22.48 \pm 2.39$ & $30.10 \pm 7.93$ & $10.38 \pm 1.49$ \\
\hline 15 & $18.30 \pm 3.29^{*}$ & $23.85 \pm 4.28^{*}$ & $13.29 \pm 1.87^{*}$ \\
\hline 30 & $17.39 \pm 2.99^{* *}$ & $21.97 \pm 4.29^{* *}$ & $15.39 \pm 1.93^{*}$ \\
\hline 60 & $17.01 \pm 1.48^{* *}$ & $19.11 \pm 1.99^{* *}$ & $18.93 \pm 1.22^{* *}$ \\
\hline 90 & $16.44 \pm 2.39^{* *}$ & $18.58 \pm 2.38^{* *}$ & $19.10 \pm 1.28^{* *}$ \\
\hline
\end{tabular}

By Student " $t$ ” Test: $P>0.05$ Not Significant; significant $p<0.01 *, p<0.001^{* *}$

\section{Assessment of L.B.T. scale:}

The baseline luminosity score of facial skin was $3.59 \pm 0.49$ which gradually significantly $(\mathrm{p}<0.001)$ increased to $6.01 \pm 0.22$. The significant improvement in skin luminosity was evident from day $15(\mathrm{p}<0.05)$.

The baseline brightness score of facial skin was $4.10 \pm$ 0.23 which gradually significantly $(\mathrm{p}<0.001)$ decreased to $2.45 \pm$
Assessment subjective efficacy parameters using $10 \mathrm{~cm}$ VAS for the improvements in skin complexion and evenness by investigator:

There was significant $(\mathrm{p}<0.05)$ decrease in VAS score for day 30 to day 60. There was significant reduction in MASI score $(\mathrm{p}<0.001)$ at day 90. VAS for the improvements in skin complexion and evenness by investigator were assessing from baseline to end of the study and the results are depicted in table 3 .

Table 3: Assessment subjective efficacy parameters using $10 \mathrm{~cm}$ VAS for the improvements in skin complexion and evenness by investigator

\begin{tabular}{|l|l|}
\hline Duration (Days) & VAS score \\
\hline Baseline & $7.87 \pm 2.55$ \\
\hline 15 & $6.10 \pm 1.89$ \\
\hline 30 & $5.76 \pm 1.17^{*}$ \\
\hline 60 & $4.13 \pm 1.96^{*}$ \\
\hline 90 & $3.22 \pm 1.43^{* *}$ \\
\hline
\end{tabular}

By Student " $t$ " Test: $P>0.05$ Not Significant; significant $p<$ $0.01 *, p<0.001 * *$

Assessment of C.L.B.T. imperfections scale:

Red discoloration to skin was significantly reduced after treatment $(\mathrm{p}<0.05)$ at day 15 . The reduction was more significant $(\mathrm{p}<0.001)$ at day 30, 60 and 90 .

Olive discoloration to skin was significantly reduced after treatment $(\mathrm{p}<0.05)$ at day 15 . The reduction was more significant $(\mathrm{p}<0.001)$ at day 30,60 and 90.

Beige coloration was indicative of skin complexion evenness the changes after treatment were measured as change in $\%$ saturation from $50 \%$. It was observed that there was significant increase in beige coloration at day $15(\mathrm{p}<0.05)$ and continued to be improved from day 30 to day 90 with significance $p<0.001$. Color imperfections scale were assess from baseline to end of the study. The results are depicted in Table 4.

Table 5: Assessment of L.B.T. scale

\begin{tabular}{|l|l|l|l|}
\hline \multirow{2}{*}{ Duration (Days) } & \multicolumn{2}{|l|}{ LBT SCORE Mean \pm SD $(\mathbf{N}=\mathbf{5 0})$} & Transparency Score \\
\cline { 2 - 4 } & Luminosity Score & Brightness Score & $6.19 \pm 1.49$ \\
\hline Baseline & $3.59 \pm 0.49$ & $4.10 \pm 0.23$ & $5.87 \pm 1.87^{*}$ \\
\hline 15 & $4.45 \pm 0.47^{*}$ & $3.29 \pm 0.48^{*}$ & $5.90 \pm 1.93$ \\
\hline 30 & $5.39 \pm 1.39^{*}$ & $3.10 \pm 1.59^{*}$ & \\
\hline
\end{tabular}

0.50 showing improved brightness of the skin. The significant improvement in skin brightness was evident from day $15(\mathrm{p}<0.05)$.

The baseline transparency score of facial skin was $6.19 \pm$ 1.49 which gradually significantly $(\mathrm{p}<0.001)$ decreased to $4.89 \pm$ 1.28 showing improved transparency of the skin. The significant improvement in skin transparency was evident from day 15 $(\mathrm{p}<0.05)$. L.B.T. scale was assessing from baseline to end of the study. The results are depicted in Table 5. 


\begin{tabular}{|l|l|l|l|}
\hline 60 & $5.89 \pm 0.48^{*}$ & $2.89 \pm 0.10^{*}$ & $5.29 \pm 1.22^{*}$ \\
\hline 90 & $6.01 \pm 0.22^{* *}$ & $2.45 \pm 0.50^{* *}$ & $4.89 \pm 1.28^{* *}$ \\
\hline
\end{tabular}

By Student " $t$ " Test: $P>0.05$ Not Significant; significant $p<0.01 *, p<0.001^{* *}$

Assessment of Global imperfection score by C.L.B.T. scale:

There was no significant $(\mathrm{p}>0.05)$ difference at day 15 .

At day 30 and 60 there was significant $(\mathrm{p}<0.01)$ and at day 90 $\mathrm{p}<0.001$ reduction in mean global imperfection score.

Global imperfection score by C.L.B.T. scale was assessing from baseline to end of the study. The results are depicted in table 6 .

Table 6: Assessment of Global imperfection score by C.L.B.T. scale

\begin{tabular}{|l|l|}
\hline Duration (Days) & $\begin{array}{l}\text { Global imperfection score (Out of 45) } \\
\text { Mean } \pm \text { SD }\end{array}$ \\
\hline Baseline & $30.28 \pm 2.19$ \\
\hline 15 & $28.10 \pm 2.99$ \\
\hline 30 & $22.19 \pm 3.29^{*}$ \\
\hline 60 & $19.38 \pm 2.29^{* *}$ \\
\hline 90 & $17.38 \pm 1.10^{* *}$ \\
\hline
\end{tabular}

By Student " $t$ " Test: $P>0.05$ Not Significant; significant $p<$ $0.05 *, p<0.001 * *$

Assessment of Digestive Behaviour Score:

There was non- significant difference in mean score of heartburn, post prandial fullness, stool consistency, bloating and constipation from baseline till end of study.

Assessment of Subject's self-assessment of skin questionnaire:
Q.1: there was significant $(\mathrm{p}<0.001)$ improvement in radiance reported by subjects from day 15 till day 90 .

Q.2: there was significant $(\mathrm{p}<0.001)$ improvement in freshness of complexion reported by subjects from day 15 till day 90 .

Q.3: there was significant $(\mathrm{p}<0.05)$ improvement in luminescence reported by subjects from day 30 till day 90 .

Q.4: there was significant $(\mathrm{p}<0.05)$ improvement in homogeneity of complexion reported by subjects from day 30 till day 90 .

Q.5: there was significant $(\mathrm{p}<0.05)$ improvement in skin smoothness reported by subjects from day 15 till day 90 .

Q.6: there was significant $(\mathrm{p}<0.05)$ improvement in refined skin texture reported by subjects from day 15 till day 90 .

Q.7: there was significant $(\mathrm{p}<0.05)$ improvement in skin firmness reported by subjects from day 15 till day 90 .

Q.8: there was significant $(\mathrm{p}<0.05)$ improvement in skin hydration reported by subjects from day 15 till day 90 .

Subject's self-assessment of skin questionnaire were done. The results are depicted in table 7

Table 7: Assessment of Subject's self-assessment of skin questionnaire

\begin{tabular}{|l|l|l|l|l|l|}
\hline \multirow{2}{*}{ Assessment } & \% Population with the assessment criteria (N=30) \\
\cline { 2 - 6 } & Baseline & Day 15 & Day 30 & Day 60 & Day 90 \\
\hline $\begin{array}{l}\text { Q-1: For you, the radiance of your complexion is? } \\
(0=\text { dull }-10=\text { glowing) }\end{array}$ & $3.38 \pm 2.57$ & $6.48 \pm 2.47^{* *}$ & $7.38 \pm 2.48^{* *}$ & $8.11 \pm 2.10^{* *}$ & $8.92 \pm 3.10^{* *}$ \\
\hline $\begin{array}{l}\text { Q-2: Is your complexion fresh? } \\
(0=\text { not }-10=\text { very) }\end{array}$ & $2.83 \pm 1.22$ & $4.33 \pm 1.39^{* *}$ & $5.10 \pm 1.10^{* *}$ & $7.83 \pm 1.18^{* *}$ & $8.01 \pm 1.14^{* *}$ \\
\hline $\begin{array}{l}\text { Q-3: Is your complexion luminous? } \\
(0=\text { not }-10=\text { very) }\end{array}$ & $3.49 \pm 1.38$ & $4.10 \pm 1.10$ & $5.39 \pm 1.39^{*}$ & $5.89 \pm 1.10^{* *}$ & $7.39 \pm 1.43^{* *}$ \\
\hline $\begin{array}{l}\text { Q-4: Is your complexion homogeneous? } \\
(0=\text { not }-10=\text { very) }\end{array}$ & $4.48 \pm 1.48$ & $4.49 \pm 2.19$ & $6.49 \pm 1.83^{*}$ & $7.39 \pm 1.83^{* *}$ & $8.15 \pm 1.48^{* *}$ \\
\hline $\begin{array}{l}\text { Q-5: Is your skin smooth? } \\
(0=\text { not }-10=\text { very) }\end{array}$ & $2.45 \pm 1.38$ & $3.11 \pm 1.34$ & $4.38 \pm 1.48^{* *}$ & $5.98 \pm 1.48^{* *}$ & $7.38 \pm 1.38^{* *}$ \\
\hline $\begin{array}{l}\text { Q-6: Is your skin texture refined? } \\
(0=\text { not refined }-10=\text { very refined) }\end{array}$ & $3.43 \pm 1.48$ & $4.47 \pm 0.99^{*}$ & $6.11 \pm 1.29^{* *}$ & $7.69 \pm 1.38^{* *}$ & $9.83 \pm 1.39^{* *}$ \\
\hline $\begin{array}{l}\text { Q-7: Is your skin firm? } \\
(0=\text { soft }-10=\text { firm) }\end{array}$ & $3.48 \pm 1.38$ & $4.83 \pm 1.02^{*}$ & $6.18 \pm 1.39^{* *}$ & $7.73 \pm 1.99^{* *}$ & $7.98 \pm 1.73^{* *}$ \\
\hline $\begin{array}{l}\text { Q-8: Is your skin hydrated? } \\
(0=\text { not }-10=\text { very) }\end{array}$ & $3.58 \pm 1.88$ & $4.48 \pm 1.48^{*}$ & $6.39 \pm 1.88^{* *}$ & $8.01 \pm 1.21^{* *}$ & $8.58 \pm 1.10^{* *}$ \\
\hline
\end{tabular}

By Student " $t$ " Test: $P>0.05$ Not Significant; significant $p<0.05^{*}, p<0.001^{* *}$

\section{Profile of adverse events}

Out of 30, $10(30 \%)$ subjects reported a total of 11 adverse events during the study period. These adverse events included fever, menstrual pain, injury, body ache, dry \& irritable eyes, cold and headache. All these adverse events were mild in severity except headache which was moderate in nature. These adverse events were resolved completely after rescue medication was given. Study treatment was not stopped during these adverse events. All these adverse events were not related to the study drug.

\section{Discussion}

www.ijirms.in
Visible skin appearance is an important moderator in human social interaction. It facilitates nonverbal communication. Humans confer great concern of a beautiful, healthy, and youthful-looking skin ${ }^{[5]}$.

The skin radiance is influenced by intrinsic and extrinsic factors which can modulate skin aging.7-10 While the intrinsic aging is inevitable, extrinsic aging is the result of the interplay of external factors and human behaviours, nutritional deficiencies, alcohol, stress, and ultraviolet (UV) exposure. Both of these factors impact the oxidative stress by an overproduction of reactive oxygen species ${ }^{[6]}$. The unhealthy skin is potentially more exposed to oxidative stress, leading to exacerbated reactive oxygen species

$-$ 
production ${ }^{[7]}$. This overproduction of free radicals, which plays an important role in damaging skin cells and in addition leads to a degradation of collagen and elastin which are responsible for the skin firmness, elasticity and the vessels integrity, and hence for the good skin vascularization ${ }^{[8]}$. Moreover, cell degradation and UV exposure will lead to a heterogeneous overproduction of melanin by melanocytes, which leads to facial spots and a heterogeneous complexion ${ }^{[9]}$. To avoid such cellular damages, a reduction of the amount of secondary oxidants is the complementary step to lower the oxidative stress. Thus, nutrition and particularly dietary antioxidant active ingredients could help to protect skin from damaging. First, they can act against the oxidative stress and secondly some of them are able to impact specific biological mechanisms enhancing the skin protection and repair.

Facial hyperpigmentation and dark spots are the main concerns when the appearance and health of skin is considered. The clinical evaluation of the hyperpigmentation is done through MASI score i.e. Melasma area severity index score. It is calculated by assessment of three parameters: Area (A), darkness (D), and homogeneity $(\mathrm{H})$ of involvement where in forehead (f) constitutes $30 \%$, right malar region (rm) 30\%, left malar region (lm) $30 \%$, and chin (c) $-10 \%$.

In the present study represents reduction in the MASI score at day 15, 30, 60 and day 90 respectively. There was also respresent reduction in MASI acne score from day 30 to day 60 .

Assessment subjective efficacy parameters using $10 \mathrm{~cm}$ VAS for the improvements in skin complexion and evenness by investigator was performed lesser score denoted improvement and greater score as uneven complexion. The VAS score was decreased throughout the period of treatment.

In the present study, skin radiance evaluated by clinical scoring using the coloring, luminosity, brightness, and transparency (C.L.B.T.TM) scale and imperfections scale (dark circles, redness/rosacea, spots, and heterogeneity). The C.L.B.T.TM methodology allows assessing different descriptors of the complexion: coloring, luminosity, brightness, and transparency of facial skin. The descriptors have been defined precisely to ensure the homogeneity of the measurements ${ }^{[10]}$.

In the present study, Red and olive discoloration to skin was significantly reduced after treatment on day 15 and same flow continued on day 30,60 and 90. It was observed that there was significant increase in beige coloration significantly at day 15 and continued to be improved from day 30 to day 90 .

The significant improvement in skin luminosity was evident from day 15.

The significant improvement in skin brightness was evident from day 15.

The significant improvement in skin transparency was evident from day 15.

The mean global imperfection score at baseline was $30.28 \pm 2.19$. There was a significant reduction in mean global imperfection score at day 30 and 60 and at day 90 .

It can be concluded from the C.L.B.T. assessment that there is much reduced skin discoloration and imperfection and improved tone of skin together with improved luminescence, brightness and transparency of the skin.

Being a skincare supplement, we checked IPs effect on digestive behavior of subjects. It was revealed that there was nonsignificant difference in mean score of heartburn, post prandial fullness, stool consistency, bloating and constipation from baseline till end of study. It predicts safety and tolerance of the product.
Skin firmness and elasticity assessment was performed on subjective self-assessment questionnaire ${ }^{[11]}$. It was apparent from the questionnaire assessment that subject reported improved radiance, freshness in complexion, refinement in skin texture, firmness and hydration of the skin significantly from day 15 and continued till day 90 .

Skin luminescence, homogeneity, complexion and skin smoothness was improved gradually from day 15 to day 90 . The significance in difference was achieved from day 30 .

As per physician and subjects, all the subjects (100\%) from both the groups reported excellent tolerability to given intervention.

Out of 30, $10(30 \%)$ subjects reported a total of 11 adverse events during the study period. These adverse events included fever, menstrual pain, injury, body ache, dry \& irritable eyes, cold and headache. All these adverse events were mild in severity except headache which was moderate in nature. These adverse events were resolved completely after rescue medication was given. Study treatment was not stopped during these adverse events. All these adverse events were not related to the study drug.

Thus BP-G01 is safe and effective in promoting health and wellbeing in healthy adults.

Clinical observations indicating beneficial effects of BPG01in improving skin health are by virtue of the ingredients used in BP-G01.

\section{Conclusions}

BP-G01 is safe and effective in promoting skin health in healthy adults.

BP-G01 improved skin luminosity, brightness, radiance and complexion from baseline to day 90 . The improvement was evident from day 15 . BP-G01 promoted skin hydration and thus the firm and suppleness. The skin discoloration was reduced by the treatment of BP-G01 including dark circles, dark spots from face and neck improving overall complexion. BP-G01 promoted skin evenness and it was evident from day 15 . Subject self-assessed questionnaire also reports the freshness in the complexion with clear, hydrated and firm skin. As there are many ingredients used in formulation BP-G01 useful in preventing photo damage, the supplement can be used for preventive benefits for young woman. BP-G01 improved skin texture and softness as a virtue of reduction of imperfections and promoting hydration to skin. BP-G01 has easily digestible blend of natural plant based pea and rice proteins which help assimilate all the herbal and phytoconstituents from the herbs like (Sea buckthorn, Pearl powder's., Orange and pomegranate), and The adaptogenic blend in BP-G01 consist of Ashwagandha, Amla, Tulsi, Mulethi and Turmeric extracts. The clinical observations in improving skin health and appearance was probably by the virtue of adaptogenic and skin specific glow blend of select herbs and their actives. BP-G01 was found to be safe and effective in improving skin health and overall appearance in healthy individuals.

\section{Ethics approval and consent to participate}

Ethical Consideration: The study was initiated only after a written approval is obtained from Independent/ Institutional Ethics Committee (IEC) and subsequent registration of study on CTRI website. The study was conducted as per approved protocol and as per Good Clinical Practices guidelines. 
CTRI Registration: After getting approval from the ethics committee, the study was registered on CTRI website. Patients were enrolled in the study only after registration of study on CTRI website. CTRI/2020/06/025644-Trial Registered Prospectively. After getting approval from CTRI principle investigator proceed for taking informed consent from subjects.

\section{References}

[1] Tzellos TG, Klagas I, Vahtsevanos K, Triaridis S, Printza A, Kyrgidis A, et al. Extrinsic ageing in the human skin is associated with alterations in the expression of hyaluronic acid and its metabolizing enzymes. Exp Dermatol. 2009;18:1028-35. doi: 10.1111/j.16000625.2009.00889.x.

[2] Makrantonaki E, Zouboulis CC, German National Genome Research Network 2 The skin as a mirror of the aging process in the human organism--state of the art and results of the aging research in the German National Genome Research Network 2 (NGFN-2) Exp Gerontol. 2007;42:879-86. doi: 10.1016/j.exger.2007.07.002.

[3] Ristow M, Schmeisser S. Extending life span by increasing oxidative stress. Free Radic Biol Med. 2011;51:327-36.

doi: 10.1016/j.freeradbiomed.2011.05.010.

[4] Ristow M, Zarse K, Oberbach A, Klöting N, Birringer $\mathrm{M}$, Kiehntopf $\mathrm{M}$, et al. Antioxidants prevent health- promoting effects of physical exercise in humans. Proc Natl Acad Sci U S A. 2009;106:8665-70. doi: 10.1073/pnas.0903485106.

[5] Samson N, Fink B, Matts PJ. Visible skin condition and perception of human facial appearance. Int J Cosmet Sci. 2010;32(3):167-184.

[6] Rocquet C, Bonté F. Molecular aspects of skin ageing: Recent data. Acta Dermatoven. 2002;11(3):71-94.

[7] Kohen R. Skin antioxidants: their role in aging and in oxidative stress - new approaches for their evaluation. Biomed Pharmacother. 1999;53(4):181-192.

[8] Mélissopoulos A, Levacher C. La Peau, structure et physiologie. 2e édition. ed: TEC\&DOC / Lavoisier; 2012.

[9] Yamakoshi J, Sano A, Tokutake S, et al. Oral intake of proanthocyanidin- rich extract from grape seeds improves chloasma. Phytother Res.2004;18(11):895899.

[10] Musnier C, Piquemal P, Beau P, Pittet JC. Visual evaluation in vivo of 'complexion radiance' using the C.L.B.T. sensory methodology. Skin Res Technol. 2004;10(1):50-56.

[11] Marion Dumoulin, David Gaudout and Benoit Lemaire, Clinical effects of an oral supplement rich in antioxidants on skin radiance in women, Clinical, Cosmetic and Investigational Dermatology, 2016:9 315-324. 